\title{
An Improved Tool Wear Monitoring Method Using Local Image and Fractal Dimension of Workpiece
}

\author{
Haicheng Yu, ${ }^{1}$ Kun Wang $\mathbb{D}^{2,3}$ Ruhai Zhang, ${ }^{1}$ Xiaojun Wu, ${ }^{1}$ Yulin Tong, ${ }^{1}$ Ruiyuan Wang, \\ and Dedao $\mathrm{He}^{4}$ \\ ${ }^{1}$ Xingii Electrical Appliances Co., Ltd., Wenzhou 325021, China \\ ${ }^{2}$ Zhejiang Industry and Trade Vocational College, Wenzhou 325002, China \\ ${ }^{3}$ Hefei Institute of Physical Science, Chinese Academy of Sciences, Hefei 230031, China \\ ${ }^{4}$ Ruiming Industrial Co., Ltd., Wenzhou 325205, China \\ Correspondence should be addressed to Kun Wang; wangkunccc@163.com
}

Received 14 March 2021; Revised 7 May 2021; Accepted 18 May 2021; Published 15 June 2021

Academic Editor: Anil Kumar

Copyright ( $\odot 2021$ Haicheng Yu et al. This is an open access article distributed under the Creative Commons Attribution License, which permits unrestricted use, distribution, and reproduction in any medium, provided the original work is properly cited.

Tool wear is a key factor that dominates the surface quality and distinctly influences the generated workpiece surface texture. In order to realize accurate evaluation of the tool wear from the generated workpiece surface after machining process, a new tool wear monitoring method is developed by fractal dimension of the acquired workpiece surface digital image. A self-made simple apparatus is employed to capture the local digital images around the region of interest. In addition, a skew correction method based on local fast Fourier transformation energy is also proposed for the surface texture direction adjustment. Furthermore, the tool wear quantitative evaluation was derived based on fractal dimension utilizing its high reliability for inherent irregularity description. The proposed tool wear monitoring method has verified its feasibility as well as its effectiveness in actual milling experiments using the material of AISI 1045 in a vertical machining center. Testing results demonstrate that the proposed method was capable of tool wear condition evaluation.

\section{Introduction}

Machining tool is a major component in a manufacturing system, and its failure (tool wear and breakage) attributes up to $20 \%$ of the machine downtime [1], and the costs of tools and tool changes account for $3-12 \%$ of the total processing cost [2]. Therefore, machining tool reliability becomes a crucial important aspect in ubiquitous manufacturing which directly influences the energy consumption and production rate [3]. Conventional tool replacement strategies employ uniform time periods determined by the subjective experience of operators. But such experiential strategies will inevitably result in early replacement which leads to that only $50-80 \%$ of the tool effective life is used [4]. To enhance the manufacturing system reliability, many strategies are proposed for the machining tool condition monitoring. With ubiquitous computing, tool wear real-time monitoring emerges as a heated research area.
Tool wear is considered to be a key factor that dominates the surface quality and also a critical index to fulfill the accuracy requirements during the machining process [5]. Tool wear monitoring or estimating is usually divided into direct monitoring and indirect monitoring [6]. Direct sensing techniques of a tool wear by using microscope or charged-couple-device (CCD) camera is a traditional visionbased tool wear measurement method [7]. But this method has to stop machine and remove the tool from holder. So, it prolongs the tact time and increases the production costs. On the contrary, many tool indirect monitoring methods are proposed by modern sensors. Indirect methods are advantageous because they do not directly affect the machining process and offer high recognition accuracy under ideal conditions.

Due to its manufacturing costs advantage and real-time monitoring, indirect tool wear detection attracts the attention of many scholars. Vibration signal is the most widely 
used method for machinery condition monitoring and fault detection [8-10]. Besides, machine learning techniques also identified a promising option in various engineering application scenarios [11-13]. Recently, advances in sensing technology have led to proposals for tool condition monitoring using various signals, such as temperature, acoustic emission, cutting force, and sound. Kovac et al. developed a novel method for predicting functional lifetime in tool parts based on a tool-work thermocouple temperature with tool wear experiment [14]. Wang et al. proposed a nondestructive tool wear evaluation method by clustering energy of acoustic emission (AE) burst signals under minimal quantity lubrication cutting condition [15]. Kong et al. presented a realtime tool wear predictive model (cutting force signal) based on the integrated radial basic function-based kernel principal component analysis (KPCA_IRBF) and relevance vector machine (RVM) and verified by two different cutting experiments [16]. Ravikumar and Ramachandran performed a tool wear monitoring system using sound signals acquired during milling of aluminum alloys [17]. Due to the interference of processing conditions and limited sensing physical characteristics, multiple modalities of sensors are instrumented to measure the different aspects of tool conditions. Wang et al. presented a virtual tool wear sensing technique based on multisensory data fusion and artificial intelligence model for tool condition monitoring [18]. Rizal et al. proposed a novel approach for tool wear classification and detection in milling process using multisensor signals and Mahalanobis-Taguchi system (MTS) [19]. However, these indirect methods present a significant drawback: all these signals could be seriously affected by the inherent noise generated in industrial environments [20-23], which reduces their performance.

Milling is the process of machining using rotary cutters to remove material by advancing a cutter into a workpiece. Highly efficient milling processes are suitable for mass production and have been widely used in manufacturing. Compared with turning machines, it is difficult for measuring rotating tools wear with multiple moving axes in milling. According to related researches [24], a reliable tool condition monitoring (TCM) can largely reduce the manufacturing costs by $10-40 \%$ by reducing downtime and maximizing the usable life of milling tools.

Machined surface topographies are the final product after manufacturing and could probably be the carriers for fabrication process and functional information. Compared with conventional direct and indirect tool wear evaluation method, machined surface topographies evaluation can be performed without the need to stop the cutting process and also enjoys the merit of free noise interference. Different machined surface defects after machining with the worn tools are investigated in the previous literatures and directly affect the subsequent mechanical properties of the manufacturing components. Machining parameters, tool type, and geometry as well as workpiece properties are among the most influential parameters in the tool wear process [25]. A higher level of surface damage was generated on machined surface under condition of worn tools than new cutting tools without considering other cutting conditions. Literature found that, apart from the cutting conditions (the cutting speed, the feed rate, and the depth of cut), tool wear has primary effects on the modification of surface roughness and has drawn the attention of many researchers [26]. However, some studies obtained the opposite results when evaluating the tool wear by surface roughness. Li et al.'s study results showed that tool wear had distinct deterioration influence on the surface roughness [27]. However, in [28], authors found that the surface roughness firstly increased and then declined when flank wear varied from 0 to $0.3 \mathrm{~mm}$. Therefore, a tool wear evaluation method based on a more reliable surface topography statistical indicator is indispensable.

Inspirited by these different topography parameters tool wear condition monitoring methods, a new tool wear evaluation method is developed by fractal dimension from the acquired workpiece surface digital image. In this research, local digital images are acquired by a self-made simple apparatus. A skew correction method based on local fast Fourier transformation (FFT) energy is also proposed for the surface texture direction adjustment. The tool wear quantitative evaluation was derived based on fractal dimension from the skew corrected digital image because of its high reliability for inherent irregularity description.

The rest of the paper is organized as follows: the tool wear monitoring framework is briefly described in Section 2 . The experiment studies are presented in Section 3, as well as the model calibration. The application examples of the developed method are explained in detail in Section 4. Some concluding remarks are given in Section 5.

\section{Tool Wear Monitoring Framework}

2.1. The Proposed Tool Wear Evaluation Method. This research proposed a new tool wear monitoring method by fractal dimension from the acquired workpiece surface digital image. The flow chart of the proposed method is presented in Figure 1. Relevant details are also described below.

Step 1: place the self-made image acquisition system (described in Section 3.1.2) on a machined workpiece surface to capture the local surface images. Meanwhile, the cutting tool is taken off to evaluate the tool wear by traditional direct tool wear method (described in Section 3.1.4).

Step 2: correct the texture skew of the raw image with the proposed skew correction algorithm in Section 2.2.

Step 3: in this step, the estimating contour is determined by the similar criterion in [29]. Then, the extracted contour curve fractal dimension is calculated (described in Section 2.2). Finally, a regression model is fitted according to the fractal dimensions and the acquired tool wear based on traditional direct tool wear method (Section 3.3).

2.2. Skew Correction Algorithm. Hough transform, projection characteristics, Fourier transform, and shearlet transform are the commonly used algorithms for skew correction 


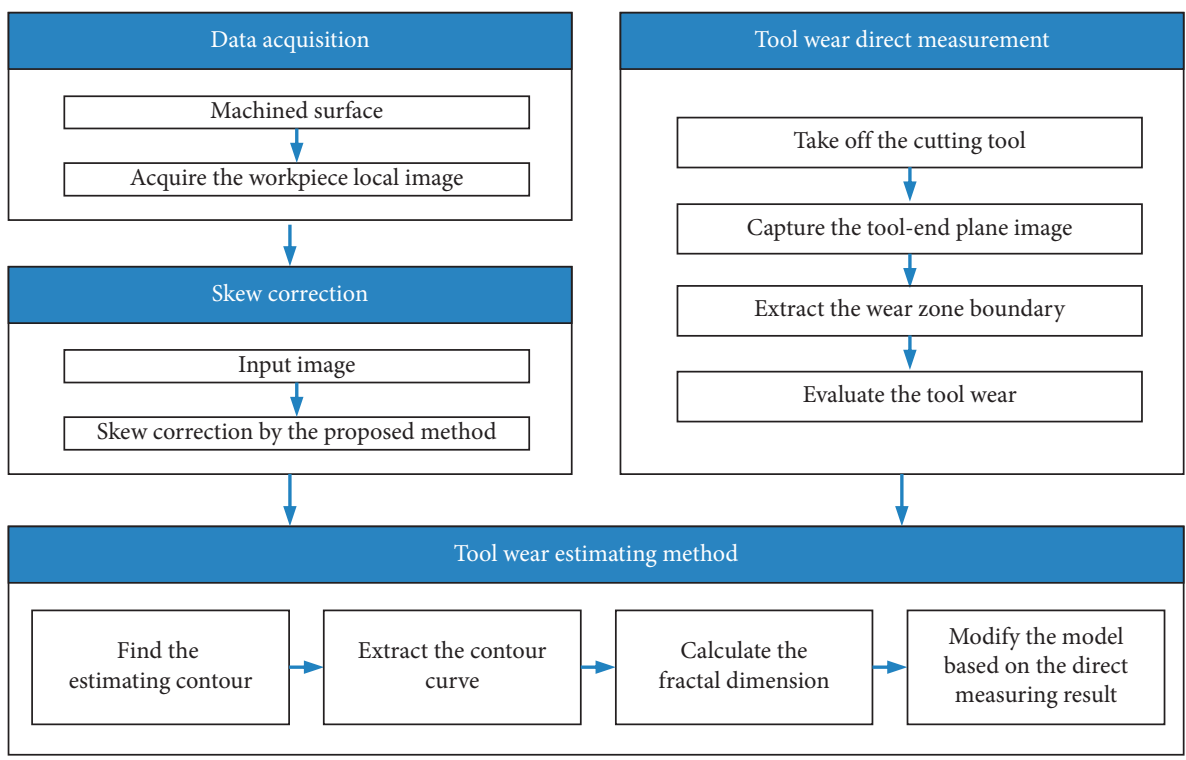

FIgURE 1: Flow chart of the proposed tool wear monitoring method.

[30-33]. Those skew detection and correction algorithms are mainly aiming at detecting the deviation of the document orientation angle from the horizontal or vertical direction. In this research, the investigated subject has a focus on the machined surface. Prior research shows that two-dimensional signal may contain strong noises in the whole frequency domain [34-36]. Therefore, a new skew correction algorithm is necessary.

2.2.1. Local Energy of the FFT. Figure 2 is a striped image with noise interference where $\lambda$ is the dominant wavelength, $\lambda_{u}$ and $\lambda_{v}$ are the wavelength components in $x$ and $y$ directions, respectively. $u=1 / \lambda_{u}$ and $v=1 / \lambda_{v}$ are the sampling rates in the two directions, and they are also the period components of the FFT spectrum $F(u, v)$. As can be seen in Figure 2, acquired surface image contains strong noises in the whole frequency domain. Therefore, the general used Fourier transform in document scanning is not suitable in this research. Notice that the wavelength of main direction ((a) in Figure 2) is slightly different from the $x$ and $y$ directions ((b) and (c) in Figure 2); it could be possible to correct the skewed images by local FFT energy distribution. Define the local FFT energy as the energy in $x$ positive half axis of Fourier spectrum $F(u, v)$. According to this definition, if the local energy reaches its maximum, the images should be well skewed.

2.2.2. Skew Correction Algorithm. In this paper, a novel skew correction algorithm based on local 2D FFT energy is proposed to improve the skew correction accuracy. Flow chart of the proposed method is presented in Figure 3 and illustrated in the following steps:

Step 1: input the original image.

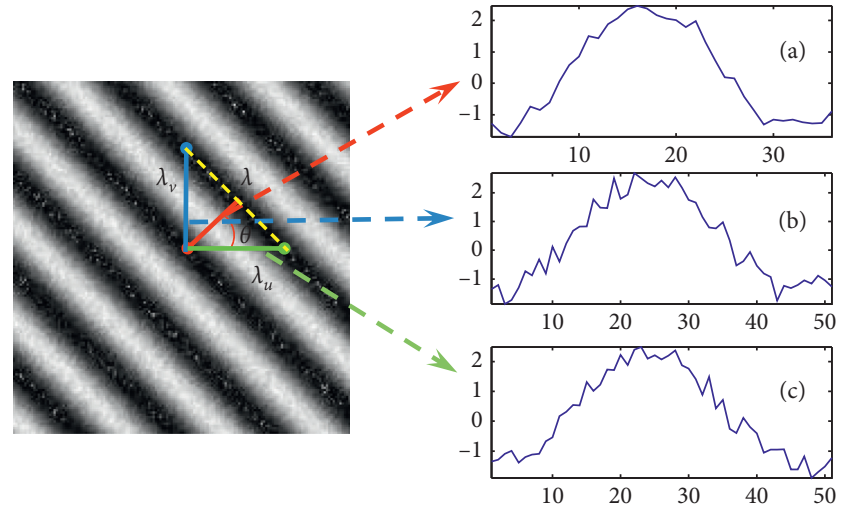

FIgURE 2: Illustration for the parameters of the 2D FFT.

Step 2: use FFT to acquire the Fourier spectrum $F(u, v)$. Step 3: calculate the local energy Li from the Fourier spectrum.

Step 4: rotate the image counterclockwise with $1^{\circ}$ and go to Step 2 until the rotate degree reach $360^{\circ}$.

Step 5: output the image which has the maximum energy $L_{i}$.

A typical captured image is shown in Figure 4(a). It can be seen that the original image has an obvious skew. This skew will strongly influence the tool wear evaluation. The local FFT energy distribution is shown in Figure 4(c) where horizon axis indicates the rotation angle and vertical axis indicates the corresponding local FFT energy. Figure 4(c) shows that the local FFT energy reaches its maximum when the rotation angle is $22^{\circ}$. Rotate the image with $20^{\circ}$; the rotated image is shown in Figure 4(b). The adjusted image result shows that the proposed method has good skew correction ability. 


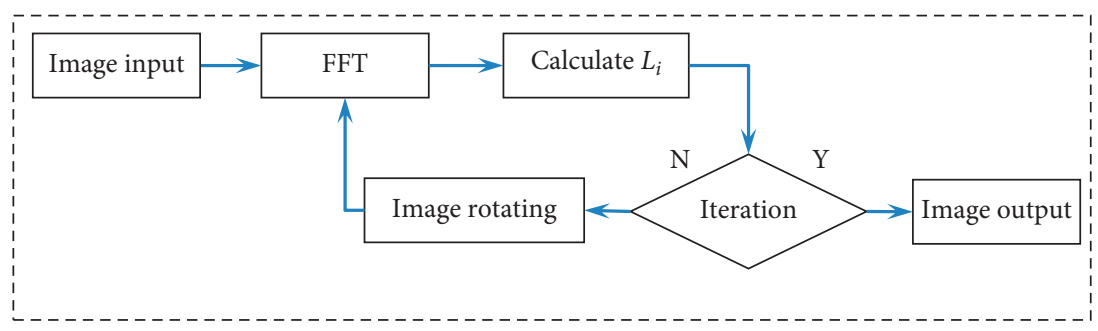

FIgURE 3: Proposed skew correction algorithm.

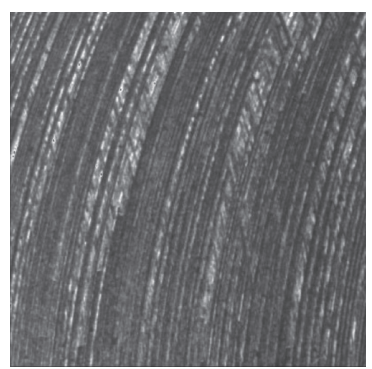

(a)

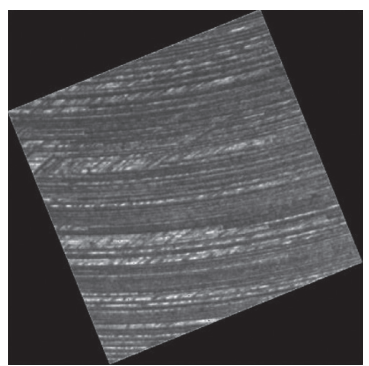

(b)

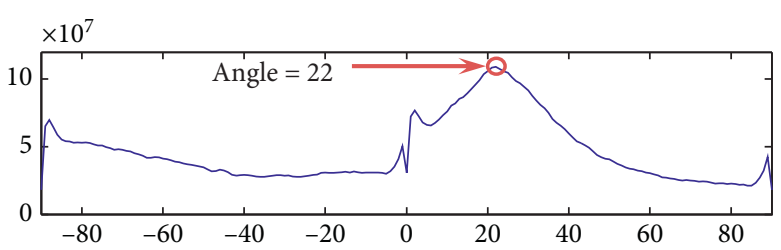

(c)

FIgURE 4: Image skew correction. (a) Original image, (b) skewed image, and (c) local energy distribution.

$$
D=\lim _{r \rightarrow 0} \frac{\log (N(r))}{\log (1 / r)},
$$

ters have been widely employed for the purpose of evaluating the tool wear from the generated workpiece. In this research, authors utilized fractal dimension as the tool wear evaluation metric.

Multifractal analysis, which is a natural extension of the fractal modeling, has aroused more attention and is applied to characterize various physical phenomena in recent years. Each geometric entity can be interpreted as a specific sort of point group. The distribution of different points on the $2 \mathrm{D}$ projection indicates different intrinsic structural topology. Generally, the distributions of $2 \mathrm{D}$ point group can be categorized into three types: even distribution, random distribution, and collectively distribution.

Fractal dimension is reported as an effective tool for describing the inherent irregularity of natural objects, as well as the point group holistic morphological characteristics [37]. The value of the fractal dimension reflects the filling ability of the point group; it is greater than the topology dimension and less than the spatial dimension [38]. The analysis of fractal patters has aroused heated discussion during the last years, mainly in the field of empirical applications [39]. The fractal dimension could be represented by several types of dimensions, for instance, the similarity dimension, Hausdorff dimension, and box-counting dimension (BCD) [40]. Among these methods, the BCD method is the most well-accepted method to determine the fractal dimension in various application fields due to its relative simplicity and reliability $[37,41]$.

Mandelbrot [42] defined that a set in an Euclidean space is said to be self-similar if it is the union of $N(r)$ distinct subsets, each of which is a copy of the original scaled down by a ratio $1 / r$ in each spatial direction. The fractal dimension of an object is defined by where $N(r)$ is the least number of boxes of length $r$ needed to completely cover the object. Based on this definition, many different dimension types are proposed. Introduced by Gangepain and Roques-Carmes [43], BCD is found to be the most simple and reliable method for approximate fractal dimension estimation. The principle of BCD is shown in Figure 5. Using a fixed rectangle to decompose the point group, the number of filling unit element will be changed with the changing of rectangle dimension $w$. By counting the length of the unit element $w$ and the filling element number $N(w)$, the fractal dimension can be approximately described with

$$
D \approx \frac{\log (N(w))}{\log (1 / w)} .
$$

Hence, the overall procedure of the BCD method can be summarized into the following steps (as can be seen in Figure 6): (a) divide the point group by boxes under different length $w$, (b) compute the number $N$ of the boxes whose number of pore pixels is bigger than 1, and (c) plot $\log (N(w))$ vs. $\log (1 / w)$ and use the linear correlation to fit the data; the slop of the generated line denotes the BCD fractal dimension [44].

As mentioned above, fractal dimension should be bigger than the topology dimension while smaller than the spatial dimension. Therefore, for the $2 \mathrm{D}$ projection, the fractal dimension is greater than 1 but less than 2 . The fractal dimension reflects the distribution evenness of the point group; large fractal dimension means good distribution evenness. Figure 5 shows three different point group 


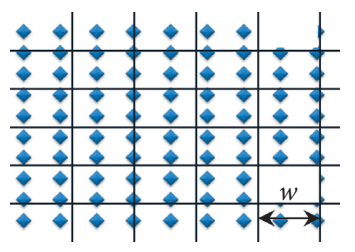

(a)

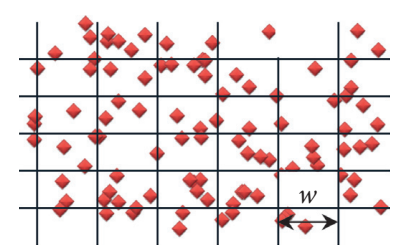

(b)

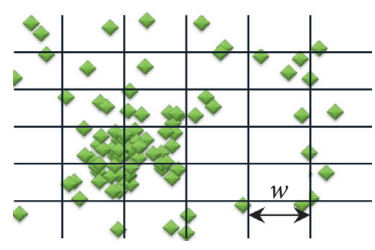

(c)

FIgURe 5: Point groups. (a) Even distribution, (b) random distribution, and (c) collective distribution.

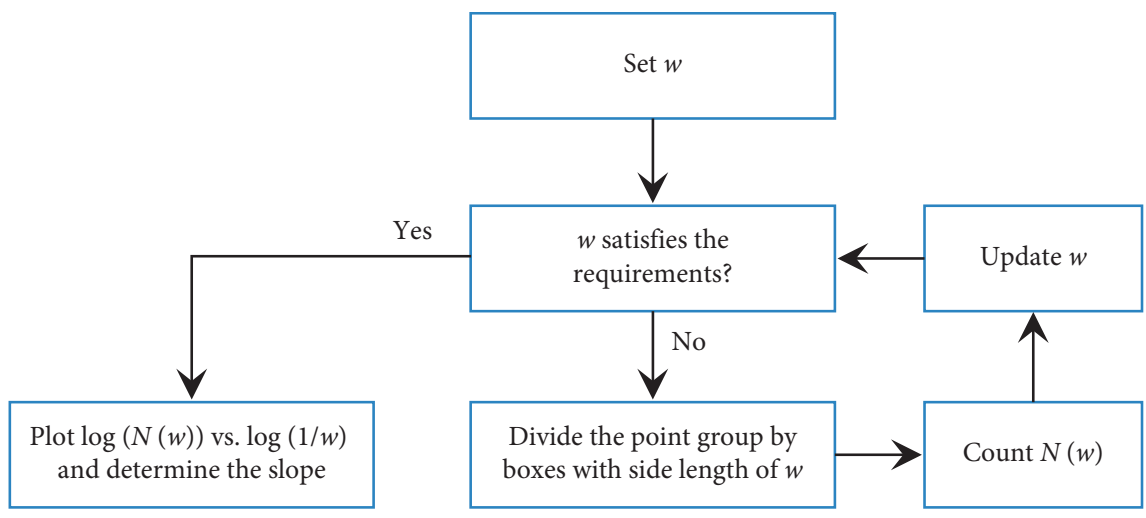

Figure 6: Flow chart of BCD method.

distribution situations. For the left blue rhombic point group, the fractal dimension is 2 , while the fractal dimension of middle and right rhombic point groups is less than 2 .

\section{Experiment Studies}

In order to investigate the proposed tool wear evaluation method, a series of AISI 1045 steel milling cutting experiments were carried out.

\subsection{Experiment Setup}

3.1.1. Workpiece and Cutting Tool. The workpiece used in this test was AISI 1045 steel, rectangular block with the dimensions of $230 \mathrm{~mm}(\mathrm{~L}) \times 90 \mathrm{~mm}(\mathrm{~W}) \times 90 \mathrm{~mm}(\mathrm{H})$. A cemented carbide end milling cutter (the diameter is $10 \mathrm{~mm}$ ) with three teeth is employed for the experiments.

3.1.2. Image Acquisition. Workpiece surface images are collected by a self-made image acquisition system; the structure sketch of the image acquisition system is shown in Figure 7. The platform is constructed of mobile phone (Xiaomi 8 , produced by Xiaomi Corporation, Beijing, China), coaxial light sources (blue light, VP-24, produced by Vanch Photoelectric Technology Co., Ltd., Shanghai, China) and other auxiliary mechanism. As can be seen in Figure 7, coaxial light has a semireflective mirror to align the light from the LED array to the same optical axis as the camera lens. Mirror surface workpieces are uniformly illuminated without unevenness. Focal length regulator is used to adjust the focal length. Focal length regulator is used to ensure the unity of focusing distance of different photos. After

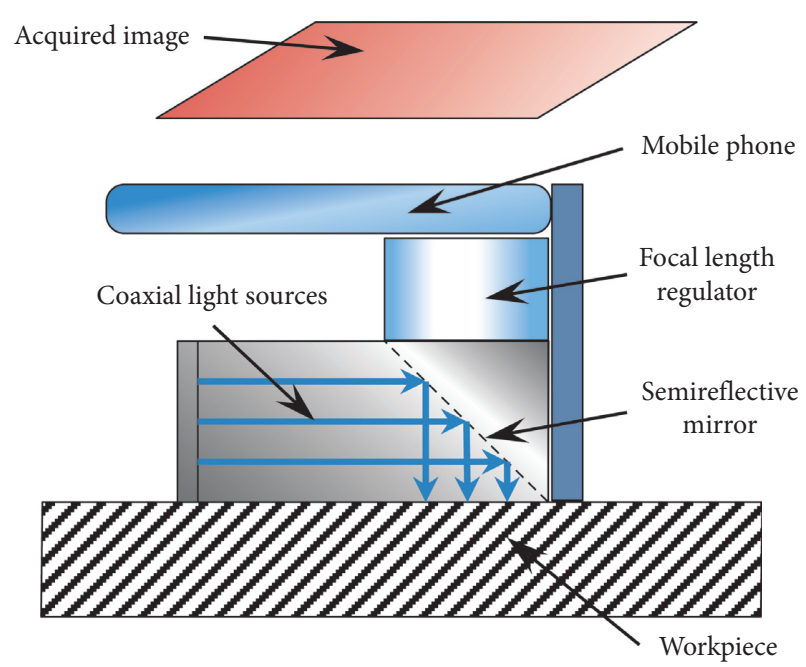

FIGURE 7: Structure sketch of the image acquisition system.

machining, the image acquisition is placed on the machined workpiece surface and captures the digital image. Details about the capture parameters are available in Table 1.

3.1.3. Experimental Parameters. The milling cutting experiments are conducted on a vertical machining center (DMTG VDL850A, produced by General technology group Dalian Machine Tool Co., Dalian, China). The experimental setup for the milling test is shown in Figure 8. The machining parameters for the milling tests are selected as spindle speed of $2000 \mathrm{rpm}$, feed rate of $600 \mathrm{~mm} / \mathrm{min}$, cutting depth of $0.5 \mathrm{~mm}$, and cutting width of $8 \mathrm{~mm}$. 
TABLE 1: Capture parameters.

\begin{tabular}{lc}
\hline Property & Information \\
\hline Exposure time & $1 / 250 \mathrm{~s}$ \\
Sensor sensitivity (ISO) & 400 \\
Equivalent focal length & $12 \mathrm{~mm}$ \\
White balance & 3200 \\
\hline
\end{tabular}

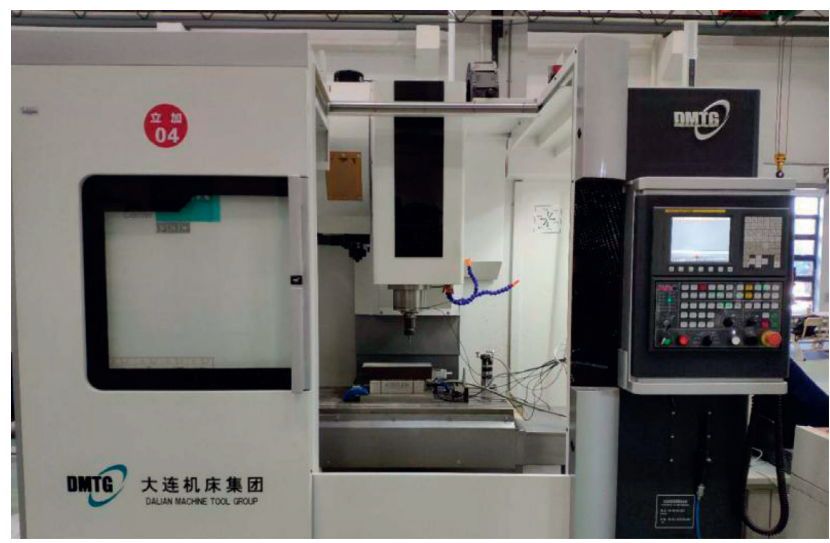

FIgURE 8: Vertical machining center.

3.1.4. Direct Wear Area Evaluation Instrument. In this experiment, the milling process is multitooth machining and each tooth is independent of each other. The corresponding average wear area is measured by an electron measuring microscope via direct measurement method (GP-300C, produced by Gaopin precision instruments Co., Ltd., Kunshan, China, as shown in Figure 9).

3.2. Evaluation Metrics. To quantitatively evaluate the performance of the developed method, different metrics are investigated including standard deviation, variance, skewness, kurtosis, roughness, and fractal dimension.

Skewness is typically used to measure the asymmetry of the probability distribution of a random variable. If skewness $S<0$, there is a negative skew which means the mass of the distribution is concentrated on the right of the distribution figure. If $S>0$, there is a positive skew which means the mass of the distribution is concentrated on the left of the distribution figure. The distribution is similar to a Gaussian normal distribution when $S=0$.

Kurtosis is a measure of whether the data are heavytailed or light-tailed relative to a Gaussian normal distribution. Data sets with high kurtosis tend to have heavy tails, or outliers. Data sets with low kurtosis tend to have light tails, or lack of outliers.

Surface roughness is a typical parameter which refers to the amplitude information and high frequency irregularities. There are many different roughness parameters in practice, but $\mathrm{Ra}$ (the arithmetical mean of the deviations) is by far the most commonly used one. $\mathrm{Ra}$ is defined as

$$
\mathrm{Ra}=\frac{1}{L} \int_{0}^{l}|z| \mathrm{d} \mathrm{l},
$$

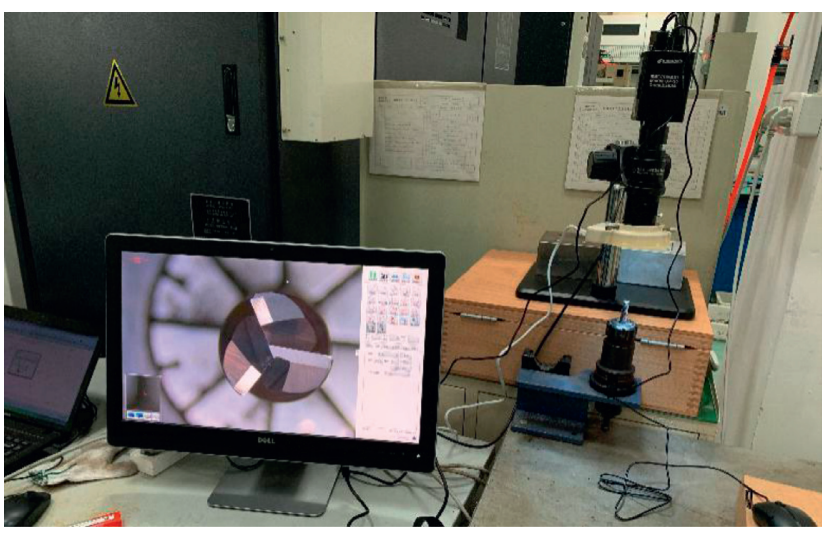

Figure 9: Direct tool wear detection.

where $L$ is the length and $z$ denotes the height. According to this definition, the surface roughness can quantify the deviations in the direction of the normal vector of a real surface from its ideal form [39].

\subsection{Model Calibration}

3.3.1. Tool Wear Evaluation by Direct Method. Some microscopic images of the employed tool are shown in Figure 10. After tool wear calculation from the acquired images, the tool wear progression with different processing layers is shown in Table 2 (direction measurement method). It has been found that the tool wear area deteriorates with the increase of processing time. According to Table 3, the tool wear process can be obviously divided into two stages, the stable wear stage and the sharp wear stage. In the stable wear stage, the mean tool wear area gradually increases from $0.023 \mathrm{~mm}^{2}$ to $0.088 \mathrm{~mm}^{2}$. In the sharp wear stage, the mean tool wear area rapidly deteriorates from $0.088 \mathrm{~mm}^{2}$ to $0.529 \mathrm{~mm}^{2}$.

3.3.2. Tool Wear Evaluation by Different Metrics. Surface images were collected from the milled AISI 1045 steel workpiece surface via the self-made image acquisition system. In the experiment, 3 images were acquired for each layer. Some typical samples of images are displayed in Figure 11. As can be seen in Figure 11, the image texture becomes blurred with the worsening of tool wear. Therefore, we can further explore the relationship between tool wear and surface image texture by appropriate statistics.

In this research, various metrics (standard deviation, variance, skewness, kurtosis, and fractal dimension) are investigated according to the acquired workpiece local images. According to the instruction of ISO 4288, the workpiece shall be properly positioned so that the direction of the section corresponds to the maximum value of the roughness. Therefore, in this research, metrics are calculated along the red dashed lines in Figure 11. Besides, a mobile roughness measuring device (type: MarSurf PS10 (produced by Mahr (Gottingen, Germany)), Figure 12) is engaged to measure the machined surface roughness by averaging the roughness values of repeated measurements. The roughness 

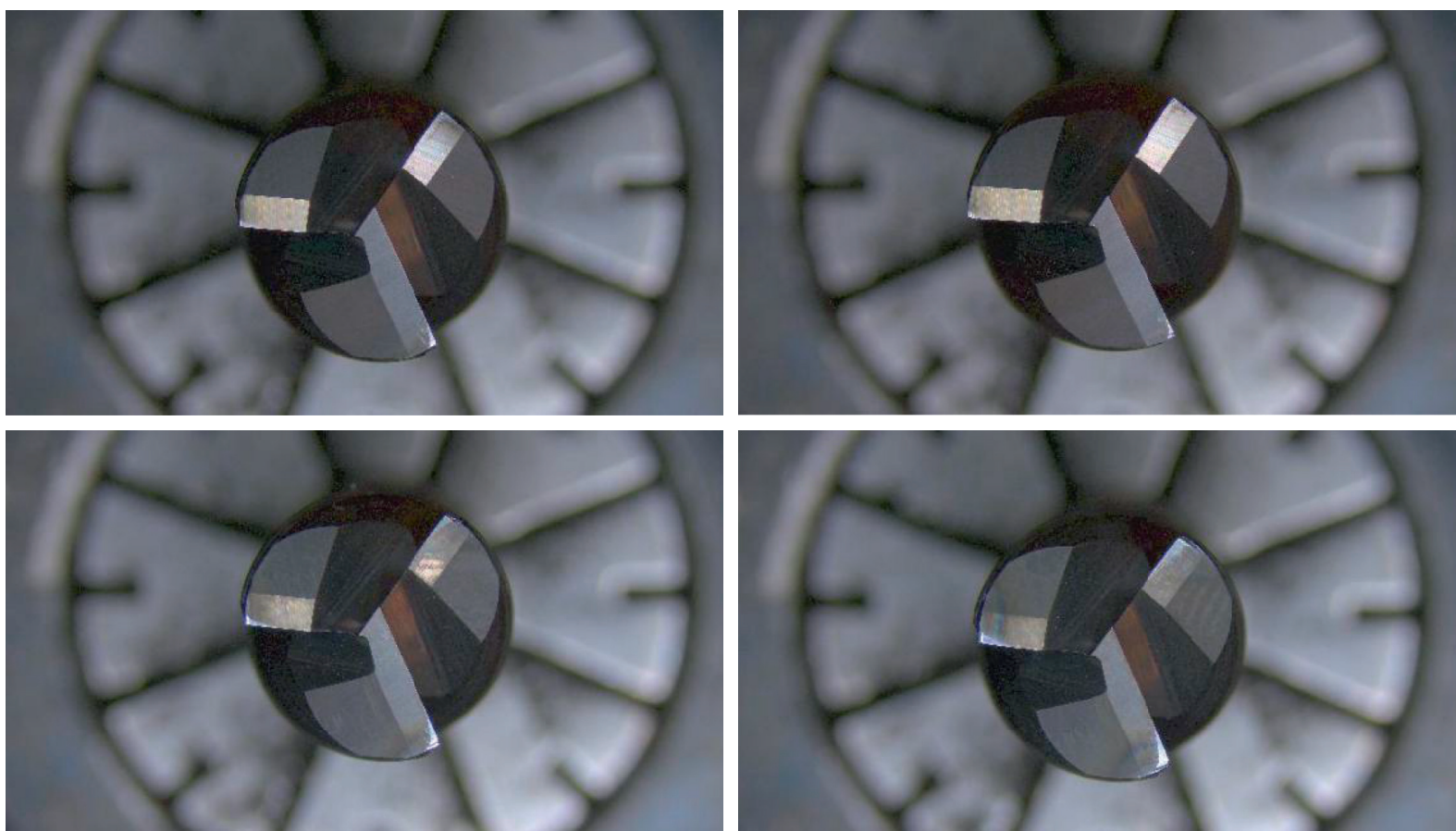

FIgURe 10: Acquired microscopic images.

TABLE 2: Tool wear areas.

\begin{tabular}{lcccc}
\hline Processing layer & Tooth 1\# wear area & Tooth 2\# wear area & Tooth 3\# wear area & Mean wear area \\
\hline 1 & 0.0266 & 0.0192 & 0.0237 & 0.023167 \\
2 & 0.0533 & 0.0577 & 0.0533 & 0.054767 \\
3 & 0.1109 & 0.0695 & 0.0843 & 0.088233 \\
4 & 0.1598 & 0.1169 & 0.4423 & 0.239667 \\
5 & 0.2604 & 0.3417 & 0.5044 & 0.368833 \\
6 & 0.716 & 0.3698 & 0.5 & 0.5286 \\
\hline
\end{tabular}

TABLE 3: Experimental parameters and results.

\begin{tabular}{lccc}
\hline No. & 1 & 2 & 3 \\
\hline Spindle speed & $1600 \mathrm{rpm}$ & $1600 \mathrm{rpm}$ & $1600 \mathrm{rpm}$ \\
Feed speed & $400 \mathrm{~mm} / \mathrm{min}$ & $500 \mathrm{~mm} / \mathrm{min}$ & $600 \mathrm{~mm} / \mathrm{min}$ \\
Cutting depth & $0.5 \mathrm{~mm}$ & $0.7 \mathrm{~mm}$ & $0.9 \mathrm{~mm}$ \\
Cutting width & $8 \mathrm{~mm}$ & $8 \mathrm{~mm}$ & $8 \mathrm{~mm}$ \\
Evaluation layer & 2 & 4 & 1 \\
Fractal dimension & 0.3374 & 0.3520 & 0.0286 \\
Tool wear area (proposed method) & 0.0234 & 0.1499 & 0.0217 \\
Tool wear area (direct method) & 0.019 & 0.148 & 0.019 \\
Error & $23.16 \%$ & $1.28 \%$ & $14.21 \%$ \\
\hline
\end{tabular}

Ra during different processing layers is shown in Table 4 . According to the recorded table, the new tool produces a large roughness and decreases soon. However, with further deterioration of tool wear, the corresponding surface roughness also increases.

Figure 13 shows all the metrics change in different processing layers. As can be seen in the figure, there is no obvious correlation between all statistical parameters and the number of cutting layers. It looks more like a random walk. To measure their similarity quantitatively, authors use the following correlation coefficient:

$$
\operatorname{Corr}\left\langle M_{i}, w_{a}\right\rangle=\frac{\sum\left(M_{i}-\overline{M_{i}}\right)\left(w_{a}-\overline{w_{a}}\right)}{\sqrt{\sum\left(M_{i}-\overline{M_{i}}\right)^{2} \sum\left(w_{a}-\overline{w_{a}}\right)^{2}}}
$$

where $M_{i}$ is the $i$-th evaluation metric and $w_{a}$ is the tool wear area. 

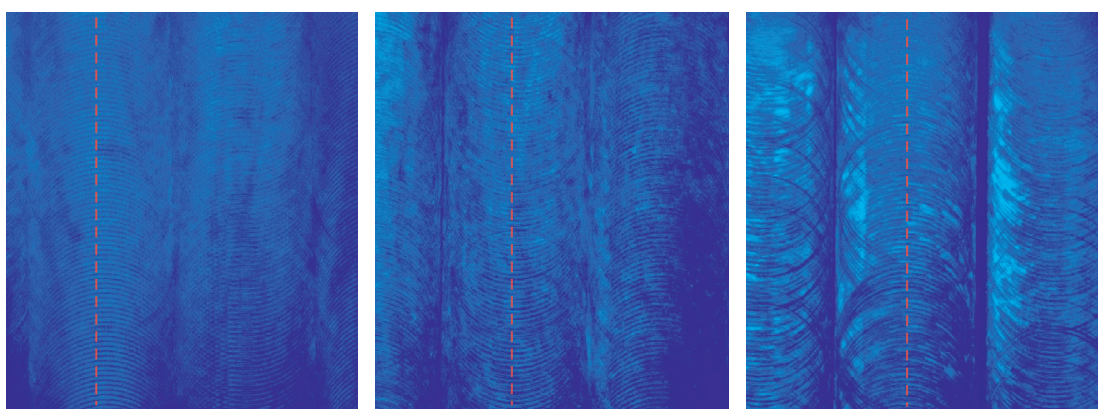

FIGURE 11: Typical samples of acquired images.

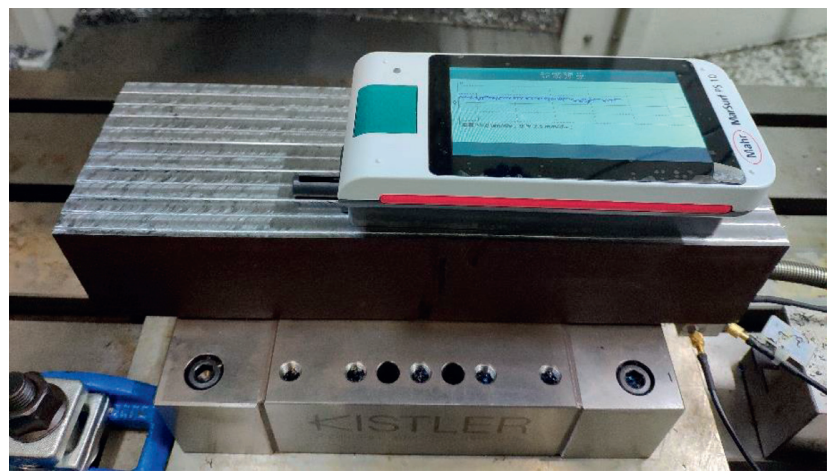

FIgURE 12: Typical samples of acquired images.

TABle 4: Capture parameters.

\begin{tabular}{llllr}
\hline Processing layer & Ra 1\# & Ra 2\# & Ra 3\# & Mean Ra \\
\hline 1 & 5.031 & 4.761 & 5.634 & 5.142 \\
2 & 2.217 & 2.359 & 1.962 & 2.179333 \\
3 & 2.467 & 2.795 & 2.464 & 2.575333 \\
4 & 2.882 & 2.293 & 1.838 & 2.337667 \\
5 & 3.314 & 4.014 & 4.481 & 3.936333 \\
6 & 5.097 & 5.139 & 5.169 & 5.135 \\
\hline
\end{tabular}

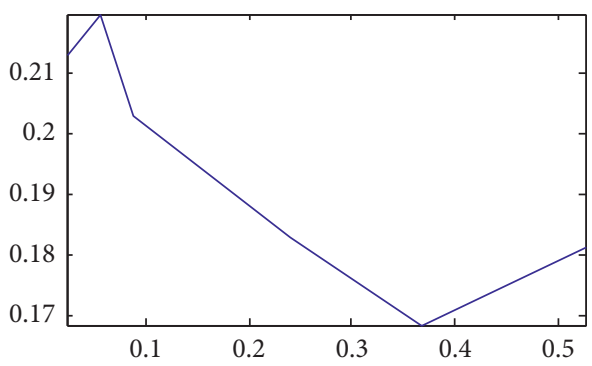

(a)

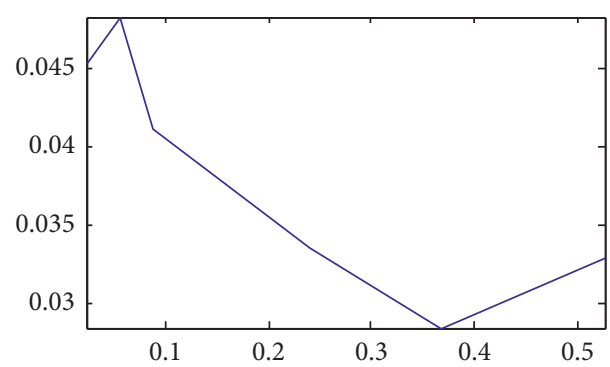

(b)

Figure 13: Continued. 


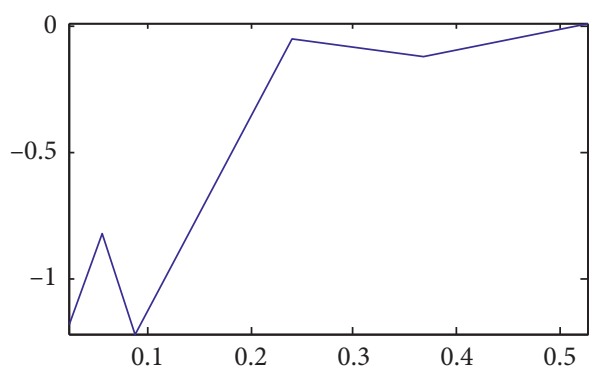

(c)

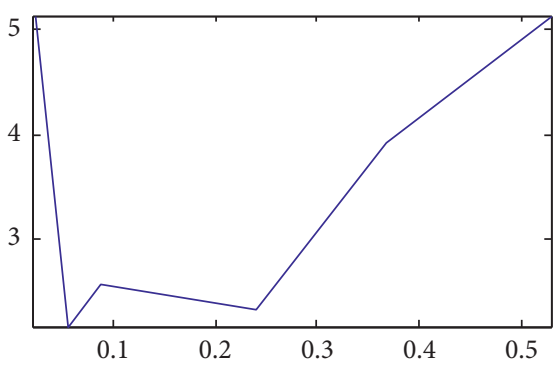

(e)

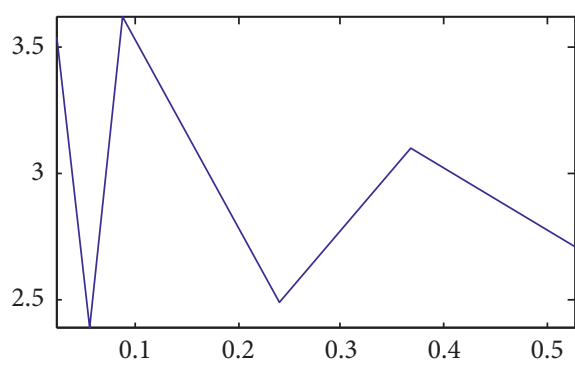

(d)

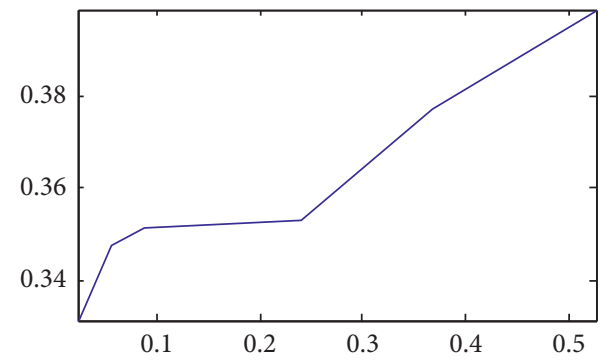

(f)

FiguRE 13: Metrics change in different processing layer: (a) standard deviation, (b) variance, (c) skewness, (d) kurtosis, (e) roughness, and (f) fractal dimension.

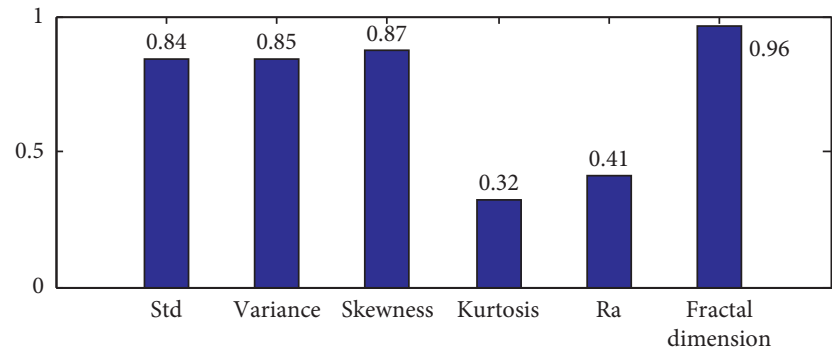

FIGURE 14: Correlation coefficients.

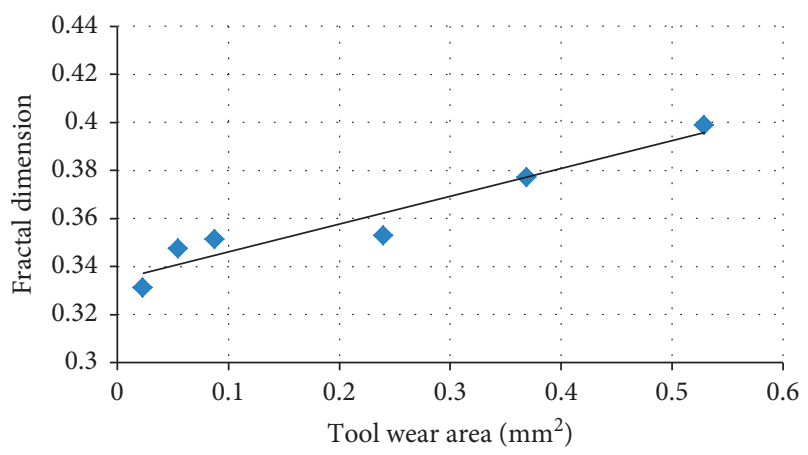

FIGURE 15: Fractal dimension change during the milling.

The correlation coefficients between the metrics and the measured tool wear areas are listed in Figure 14. It is inferred from Figure 14 that the fractal dimension strongly correlated to the tool wear area and their correlation coefficient is as high as 0.96. Based on the results, it is consolidated to conclude that fractal dimension is an effective utensil to evaluate the tool wear area.
3.3.3. Model Calibration. As presented before, tool wear area is strongly related to the fractal dimension of workpiece local image and exhibit proportional relation. Applying linear fitting to the fractal dimension (shown in Figure 15, where $x$ axis indicates the corresponding fractal dimension and $y$ axis indicates the tool wear area), the $R$ square is 0.9247 with root mean squared error (RMSE) of 0.007394, which means the linear fitting is highly significant. The fitting linear equation can be represented as

$$
F_{d}=0.1154 w_{a}+0.3347
$$

where $w_{a}$ is the tool wear area and $F_{d}$ is the fractal dimension.

As can be seen in Figure 15, it can be concluded that tool wear areas are highly proportional to fractal dimensions. As a consequence, linear fitting can efficiently reveal the relationship between tool wear area and fractal dimension, which also demonstrates that tool wear can be accurately evaluated by fractal dimension of workpiece local image.

\section{Validation Experiments of the Developed Method}

To make a quantitative analysis of the results, the measurements collected using the calibrated model are compared to those from conventional direct tool wear evaluation method in this section. The performance of the developed method was tested on 3 different milling experiments. According to the results, the values of these indicators are highly consistent with those measured via direct method. Considering the results from the direct method as the theoretical value of tool wear, the relative errors were calculated to be $23.16 \%, 1.28 \%$, and $14.21 \%$ respectively. As can 
be seen in Table 3, the proposed method offers exciting opportunities for tool wear evaluation. However, uncertainty still remains during the validation experiment, and relative error is reported as $23.16 \%$ in the first experiment and is worth further mechanistic research.

\section{Conclusion}

This paper developed a new tool wear monitoring method by fractal dimension from the acquired workpiece surface digital image. In this research, a self-made simple apparatus is employed to capture the local digital images. Then, a skew correction method based on local FFT energy is also proposed for the surface texture direction adjustment. Finally, tool wear situations are accurately evaluated by fractal dimension of workpiece local image. An actual milling experiment is performed in a vertical machining center to verify the proposed method. Testing results demonstrate the proposed method has achieved high-precision tool wear estimation. The major findings of this work can be summarized as follows:

(1) A novel tool wear monitoring method is proposed in this research where surface texture information is employed for the tool wear estimation. Compared with conventional methods, the proposed method can be performed without the need to stop the cutting process and also enjoys the merit of free noise interference.

(2) A skew correction method based on local FFT energy is also proposed for the surface texture direction adjustment. The test result shows that the proposed algorithm can effectively correct the skew in strong noises.

(3) Fractal dimension is firstly utilized for the tool wear estimation. Compared with other various metrics, fractal dimension strongly correlated to the tool wear area. The result demonstrates that tool wear can be accurately evaluated by fractal dimension of workpiece local image.

The research demonstrated that workpiece surface texture contains much tool geometrical information which can be utilized to extract the toll wear information based on the surface digital information. However, the authors believed that machined surface topographies carry far more fabrication processes and functional information and are worth further explorations.

\section{Data Availability}

The data used to support the findings of this study are included within the article.

\section{Conflicts of Interest}

The authors declare that they have no conflicts of interest.

\section{Acknowledgments}

This work was financially supported by Wenzhou Municipal Major Scientific and Technological Innovation Project (no. ZG2020023).

\section{References}

[1] L. Fernández-Robles, G. Azzopardi, E. Alegre, N. Petkov, and M. Castejón-Limas, "Identification of milling inserts in situ based on a versatile machine vision system," Journal of Manufacturing Systems, vol. 45, pp. 48-57, 2017.

[2] Y. Zhou and W. Xue, "Review of tool condition monitoring methods in milling processes," The International Journal of Advanced Manufacturing Technology, vol. 96, no. 5-8, pp. 2509-2523, 2018.

[3] Z. Lei, Y. Zhou, B. Sun, and W. Sun, "An intrinsic timescale decomposition-based kernel extreme learning machine method to detect tool wear conditions in the milling process," The International Journal of Advanced Manufacturing Technology, vol. 106, pp. 12032-21212, 2020.

[4] K. Salonitis and A. Kolios, "Reliability assessment of cutting tool life based on surrogate approximation methods," The International Journal of Advanced Manufacturing Technology, vol. 71, no. 5-8, pp. 1197-1208, 2014.

[5] Y. Zhou, B. Sun, W. Sun, and Z. Lei, "Tool wear condition monitoring based on a two-layer angle kernel extreme learning machine using sound sensor for milling process," Journal of Intelligent Manufacturing, in Press, 2020.

[6] M. Nouri, B. K. Fussell, B. L. Ziniti, and E. Linder, "Real-time tool wear monitoring in milling using a cutting condition independent method," International Journal of Machine Tools and Manufacture, vol. 89, pp. 1-13, 2015.

[7] J. Wang, Y. Zheng, P. Wang, and R. X. Gao, "A virtual sensing based augmented particle filter for tool condition prognosis," Journal of Manufacturing Processes, vol. 28, no. 3, pp. 472478, 2017.

[8] W. Sun, Y. Zhou, J. Xiang, B. Chen, and W. Feng, "Hankel matrix-based condition monitoring of rolling element bearings: an enhanced framework for time-series analysis," IEEE Transactions on Instrumentation and Measurement, vol. 70, Article ID 3512310, 2021.

[9] W. Sun, Y. Zhou, X. Cao, B. Chen, W. Feng, and L. Chen, “A two-stage method for bearing fault detection using graph similarity evaluation," Measurement, vol. 165, Article ID 108138, 2020.

[10] A. Kumar, Y. Zhou, C. P. Gandhi, R. Kumar, and J. Xiang, "Bearing defect size assessment using wavelet transform based Deep Convolutional Neural Network (DCNN)," Alexandria Engineering Journal, vol. 59, no. 2, pp. 999-1012, 2020.

[11] A. Kumar, C. P. Gandhi, Y. Zhou, G. Vashishtha, R. Kumar, and J. Xiang, "Improved CNN for the diagnosis of engine defects of 2-wheeler vehicle using wavelet synchro-squeezed transform (WSST)," Knowledge-Based Systems, vol. 285, Article ID 106453, 2020.

[12] A. Kumar and R. Kumar, “"Least square fitting for adaptive wavelet generation and automatic prediction of defect size in the bearing using levenberg-marquardt backpropagation," Journal of Nondestructive Evaluation, vol. 36, Article ID 7, 2017.

[13] G. Vashishtha and R. Kumar, "An effective health indicator for pelton wheel using levy flight mutated genetic," Algorithm" Measurement Science and Technology, in Press, 2021.

[14] P. Kovac, M. Gostimirovic, D. Rodic, and B. Savkovic, "Using the temperature method for the prediction of tool life in sustainable production," Measurement, vol. 133, pp. 320-327, 2019.

[15] C. Wang, Z. Bao, P. Zhang, W. Ming, and M. Chen, "Tool wear evaluation under minimum quantity lubrication by 
clustering energy of acoustic emission burst signals," Measurement, vol. 138, pp. 256-265, 2019.

[16] D. Kong, Y. Chen, N. Li, C. Duan, L. Lu, and D. Chen, "Relevance vector machine for tool wear prediction," $\mathrm{Me}$ chanical Systems and Signal Processing, vol. 127, pp. 573-594, 2019.

[17] S. Ravikumar and K. I. Ramachandran, "Tool wear monitoring of multipoint cutting tool using sound signal features signals with machine learning techniques," Materials Today: Proceedings, vol. 5, no. 11, pp. 25720-25729, 2018.

[18] J. Wang, J. Xie, R. Zhao, L. Zhang, and L. Duan, "Multisensory fusion based virtual tool wear sensing for ubiquitous manufacturing," Robotics and Computer-Integrated Manufacturing, vol. 45, pp. 47-58, 2017.

[19] M. Rizal, J. A. Ghani, M. Z. Nuawi, and C. H. C. Haron, "Cutting tool wear classification and detection using multisensor signals and Mahalanobis-Taguchi System," Wear, vol. 376-377, pp. 1759-1765, 2017.

[20] J. Huang, B. Chen, Y. Li, and W. Sun, "Fractal geometry of wavelet decomposition in mechanical signature analysis," Measurement, vol. 173, Article ID 108571, 2020.

[21] B. Chen, Y. Li, X. Cao, W. Sun, and W. He, "Removal of power line interference from ECG signals using adaptive notch filters of sharp resolution," IEEE Access, vol. 7, pp. 150667-150676, 2019.

[22] A. Kumar and R. Kumar, "Adaptive artificial intelligence for automatic identification of defect in the angular contact bearing," Neural Computing and Applications, vol. 29, no. 8, pp. 277-287, 2018.

[23] G. Vashishtha, S. Chauhan, M. Singh, and R. Kumar, "Bearing defect identification by swarm decomposition considering permutation entropy measure and opposition-based slime mould algorithm," Measurement, vol. 178, Article ID 109389, 2021.

[24] A. G. Rehorn, J. Jiang, and P. E. Orban, "State-of-the-art methods and results in tool condition monitoring: a review," The International Journal of Advanced Manufacturing Technology, vol. 26, no. 7-8, pp. 693-710, 2005.

[25] M. Klaic, Z. Murat, T. Staroveski, and D. Brezak, "Tool wear monitoring in rock drilling applications using vibration signals," Wear, vol. 408-409, pp. 222-227, 2018.

[26] X. Liang, Z. Liu, and B. Wang, "State-of-the-art of surface integrity induced by tool wear effects in machining process of titanium and nickel alloys: a review," Measurement, vol. 132, pp. 150-181, 2019.

[27] M. Li, S. L. Soo, D. K. Aspinwall, D. Pearson, and W. Leahy, "Study on tool wear and workpiece surface integrity following drilling of CFRP laminates with variable feed rate strategy," Procedia CIRP, vol. 71, pp. 407-412, 2018.

[28] X. Liang and Z. Liu, "Tool wear behaviors and corresponding machined surface topography during high-speed machining of Ti-6Al-4V with fine grain tools," Tribology International, vol. 121, pp. 321-332, 2018.

[29] W. Sun, B. Yao, B. Chen et al., "Noncontact surface roughness estimation using 2D complex wavelet enhanced ResNet for intelligent evaluation of milled metal surface quality," Applied Sciences, vol. 8, Article ID 381, 2020.

[30] R. N. Verma and L. G. Malik, "Review of illumination and skew correction techniques for scanned documents," Procedia Computer Science, vol. 45, pp. 322-327, 2015.

[31] F. Zhang, Y. Zhang, X. Qu, B. Liu, and R. Zhang, "Scanned document images skew correction based on shearlet transform," Multi-disciplinary Trends in Artificial Intelligence, vol. 9426, 2015.
[32] W. Li, M. Breier, and D. Merhof, "Skew correction and line extraction in binarized printed text images," in Proceedings of the IEEE International Conference on Image Processing (ICIP), pp. 472-476, Quebec, Canada, September 2015.

[33] M. Shafii and M. Sid-Ahmed, "Skew detection and correction based on an axes-parallel bounding box," International Journal on Document Analysis and Recognition (IJDAR), vol. 18, no. 1, pp. 59-71, 2015.

[34] W. Sun, X. Cao, B. Chen, Y. Zhou, Z. Shen, and J. Xiang, "A two-stage vision-based method for measuring the key parameters of ball screws," Precision Engineering, vol. 66, pp. 76-86, 2020.

[35] W. Sun and X. Cao, "Curvature enhanced bearing fault diagnosis method using 2D vibration signal," Journal of Mechanical Science and Technology, vol. 34, no. 6, pp. 2257-2266, 2020.

[36] W. Sun, N. Zeng, and Y. He, "Morphological arrhythmia automated diagnosis method using gray-level Co-occurrence matrix enhanced convolutional neural network," IEEE Access, vol. 7, pp. 67123-67129, 2019.

[37] H.-R. So, G.-B. So, and G.-G. Jin, "Enhancement of the BoxCounting Algorithm for fractal dimension estimation," Journal of Institute of Control, Robotics and Systems, vol. 22, no. 9, pp. 710-715, 2016.

[38] H. I. Tijani, N. Abdullah, A. Yuzir, and Z. Ujang, "Rheological and fractal hydrodynamics of aerobic granules," Bioresource Technology, vol. 186, pp. 276-285, 2015.

[39] K. Deng, D. Pan, X. Li, and F. Yin, "Spark testing to measure carbon content in carbon steels based on fractal box counting," Measurement, vol. 133, pp. 77-80, 2019.

[40] Y. Xu, R. Zhang, S. Ma, X. Yang, and F. Wang, "Fractal dimension of concrete meso-structure based on X-ray computed tomography," Powder Technol, vol. 350, pp. 91-99, 2019.

[41] J. Yan, Y. Sun, S. Cai, and X. Hu, "An improved box-counting method to estimate fractal dimension of images," Journal of Applied Analysis \& Computation, vol. 6, no. 4, pp. 1114-1125, 2016.

[42] B. Mandelbrot, "How long is the coast of Britain? Statistical self-similarity and fractional dimension," Science, vol. 156, no. 3775, pp. 636-638, 1967.

[43] J. J. Gagnepain and C. Roques-Carmes, "Fractal approach to two-dimensional and three-dimensional surface roughness," Wear, vol. 109, no. 1-4, pp. 119-126, 1986.

[44] H. Wu, Y. Zhou, Y. Yao, and $\mathrm{K}$. Wu, "Imaged based fractal characterization of micro-fracture structure in coal," Fuel, vol. 239, pp. 53-62, 2019. 